\title{
ON SCATTERED SPACES ${ }^{i}$
}

\author{
V. KANNAN AND M. RAJAGOPALAN
}

\begin{abstract}
We show that each 0-dimensional Hausdorff space which is scattered can be mapped continuously in a one-to-one way onto a scattered 0-dimensional Hausdorff space of the same weight as its cardinality. This gives an easier and a new proof of the fact that a countable regular space admits a coarser compact Hausdorff topology if and only if it is scattered. We also show that a $\mathbf{0 -}$ dimensional, Lindelöf, scattered first-countable Hausdorff space admits a scattered compactification. In particular we give a more direct proof than that of Knaster, Urbanik and Belnov of the fact that a countable scattered metric space is a subspace of $[1, \Omega)$, and deduce a result of $\mathrm{W}$. H. Young as a corollary.
\end{abstract}

1. A problem of Banach is to characterise all topological spaces which admit a weaker compact Hausdorff topology. Katetov [3] gave a partial solution to this problem by showing that among countable spaces they are precisely the scattered spaces. His approach does not throw enough light on the cardinal invariants attached to such a space. We prove a theorem below, in the second section, a relation between scatteredness and weights of topological spaces. This gives a new proof of Katetov's theorem mentioned above. This relates to a study of scatteredness and the problem of existence of scattered compactifications of scattered completely regular spaces. Many mathematicians are interested in this problem (to mention one see Semadeni [9] and Ryll-Nardzewski and Telgarsky [8]). In the first section of this paper we show that a scattered, 0-dimensional, first-countable, Hausdorff Lindelöf space admits a Hausdorff scattered compactification. This can be achieved by an application of the results of Knaster-Urbanik [4] and Mazurkiewicz-Sierpinski [5]. However our proof is elementary.

Definition 1. A topological space $X$ is called scattered if given a nonempty set $A \subset X$ there is an element $x_{0} \in A$ which is isolated relative to $A$.

Presented to the Society, November 25, 1972; received by the editors September 22, 1972.

AMS (MOS) subject classifications (1970). Primary 54A05, 54E35, 54D20, 54D30.

Key words and phrases. Scattered space, derived length, weight, $[1, \Omega)$.

${ }^{1}$ This is a revised version of an earlier paper. The authors thank the referee for pointing out the reference [4] and Franklin for the reference [8].

(c) American Mathematical Society 1974 
Definition 2. Let $X$ be a topological space. Let $A \subset X$. We define $A^{0}=A ; A^{1}=\{x \mid x \in A$ and $x \in \operatorname{cl}(A-\{x\})\}$. If $\alpha$ is an ordinal and $A^{\alpha}$ has been defined then we put $A^{\alpha+1}=\left(A^{\alpha}\right)^{1}$. If $\alpha$ is a limit ordinal and $A^{\beta}$ has been defined for all ordinals $\beta<\alpha$ then we put $A^{\alpha}=\bigcap_{\beta<\alpha} A^{\beta}$. If $\alpha$ is an ordinal we call $A^{\alpha}$ the $\alpha$ th derivative of $A$. A topological space $X$ is said to have a derived length if $X^{\alpha}=\varnothing$ for some ordinal $\alpha$. In this case the derived length of $X$ is defined as the least ordinal $\alpha$ so that $X^{\alpha}=\varnothing$. It is noted as $\alpha(X)$.

The following theorem can be deduced from the results of [4], [5], [9] and a theorem of Young (see Corollaries 3, 4). However our approach is easier and hence we feel it worthwhile to present our proof of this result.

THEOREM 1. A first-countable 0-dimensional Lindelöf Hausdorff space $X$ is scattered if and only if it admits a scattered compactification.

Proof. If $X$ admits a scattered compactification then $X$ is clearly scattered. So we assume that $X$ is scattered and we exhibit a scattered compactification for $X$. For this we note that since $X$ is Lindelö $X^{\alpha}=\varnothing$ for a countable ordinal $\alpha$. Now it is easy to see that such a space is hereditarily Lindelöf. Now two cases arise.

Case (i). $\alpha$ is a limiting ordinal. Then $\alpha$ is a limit of an increasing sequence $\alpha_{1}<\alpha_{2}<\cdots<\alpha_{n}<\cdots$ of countable ordinals. Then each $X-X^{\alpha_{i}}$ can be written as a countable disjoint union $\bigcup_{j=1}^{\infty} V_{i j}$ of clopen sets $V_{i j}$. Putting

$$
W_{i}=\bigcup_{1 \leqq j \leqq i, 1 \leqq k \leqq i} V_{j k}
$$

we get

$$
\bigcup_{i=1}^{\infty} W_{i}=X .
$$

It is also clear that $\alpha\left(W_{i}\right) \leqq \alpha_{i} \forall i=1,2,3, \cdots$, and $X=\bigcup_{i=1}^{\infty}\left(W_{i}-W_{i-1}\right)$ where $W_{0}=\varnothing$ and each $W_{i}-W_{i-1}$ is clopen in $X$. By induction we can assume that each $W_{i}-W_{i-1}$ has a scattered compactification $K_{i}$. Then the one-point compactification of the disjoint union of the $K_{i}$ 's is easily verified to be a scattered compactification of $X$.

Case (ii). $\alpha$ is a discrete ordinal $>1$ and hence $\alpha=\beta+1$ for some ordinal $\beta$. Then $X^{\beta}$ is countable and discrete. So there exists a countable disjoint collection $V_{1}, V_{2}, \cdots, V_{n}, \cdots$ of clopen sets in $X$ so that $V_{i} \cap X^{\beta}$ is a singleton for each $i$ and $X^{\beta} \subset \bigcup_{i=1}^{\infty} V_{i}$. Now putting $V=\bigcup_{i=1}^{\infty} V_{i}$ we can separate the two disjoint closed sets $X-V$ and $X^{\beta}$ by open sets $U$ and $W$, where $W \supset X-V$. Write $W$ as a disjoint union $\bigcup_{i=1}^{\infty} W_{i}$ of clopen sets $W_{i}$ 
and put

and

$$
F_{n}=W_{n / 2}-\bigcup_{i=1}^{n / 2} V_{i} \text { if } n \text { is even }
$$

$$
F_{n}=V_{(n+1) / 2}-\bigcup_{i=1}^{(n-1) / 2} W_{i} \text { if } n \text { is odd }
$$

taking $\bigcup_{i=1}^{n-1} W_{i}=\varnothing$ when $n=1$. Then $X=\bigcup_{n=1}^{\infty} F_{n}$ and the $F_{n}$ 's form a pairwise disjoint collection of clopen sets. By the induction assumption each $F_{2 n}$ admits a scattered compactification $G_{2 n}$. Now consider some $F_{i}$ where $i$ is odd. Let $F_{i} \cap X^{\beta}=\left\{a_{i}\right\}$. Then the first axiom is satisfied at $a_{i}$. So $F_{i}$ can be written as a disjoint union $\left\{a_{i}\right\} \cup \bigcup_{k=1}^{\infty} S_{k}$ where each $S_{k} \subset F_{i}$ is clopen in $F_{i}$. By the induction assumption each $S_{k}$ admits a scattered compactification $Y_{k}$. Then taking $Y$ to be the one-point compactification of the free union of the $Y_{k}$ 's with $a_{i}$ as the point at $\infty$ we get a scattered compactification of $G_{i}$ of $F_{i}$. Then take $Y$ to be the one-point compactification of the free union of the $G_{n}$ 's where $n=1,2, \cdots$. Then $Y$ is the required compactification.

It is clear that if $\alpha=1$ then $X$ admits a scattered compactification. So the theorem is true.

COROllary 2. Every countable metric space $X$ can be written as a disjoint union $A \cup B$ of two of its subspaces where $B$ is homeomorphic to a subspace of $[1, \Omega]$ where $\Omega$ is the first uncountable ordinal and $A$ is a closed subspace which is homeomorphic to the space of all rationals or empty.

Proof. Take $A$ to be $\bigcap_{|\alpha| \leqq|X|} X^{\alpha}$ and $B=X-A$. Then $A=\varnothing$ or is homeomorphic to the rationals (see [2] and [10]). $B$ is scattered and hence admits a scattered compactification by Theorem 1 which is, in fact, a countable compactification by following the proof of Theorem 1 carefully. So by results of [5] $B$ is a subspace of $[1, \Omega]$.

Remark. The study of how $A$ and $B$ fit together to make up the space $X$ is difficult. For some study on this see [7].

Corollary 3 (KNaster and Urbanik and Belnov). For a countable space $X$ the following are equivalent:

(a) $X$ is scattered and metrizable.

(b) $X$ is a subspace of $[1, \Omega)$.

(c) $X$ admits a complete metric.

(d) X admits a countable compactification.

We omit the proof since it follows easily from Corollary 1.

Corollary 4 (Young). A scattered subset of the real line is $a G_{\delta}$. 
2. Topologies coarser than scattered topologies. It is an unsolved problem of Banach to characterise all topologies which admit a coarser compact Hausdorff topology. Katetov [3] gave a partial solution to this problem by proving that among countable regular spaces, these are precisely the scattered topologies. Here we prove two theorems concerning the weakening of scattered topologies which, together with Theorem 1, give a different proof of the result of [3].

Let $\mathscr{Z}$ denote the class of all zero-dimensional scattered Hausdorff spaces. The following theorem asserts that each member of $\mathscr{Z}$ admits a weaker topology belonging to $\mathscr{Z}$ and satisfying some more conditions.

THeORem 5. For each $X \in \mathscr{Z}$, there exists $Y \in \mathscr{Z}$ and a continuous bijection $e: X \rightarrow Y$ such that

(i) $e\left(X^{\alpha}\right)=Y^{\alpha}$ for each ordinal $\alpha$.

(ii) The weight of $Y$ does not exceed its cardinality.

Proof. Since $X$ is scattered, for each $x$ in $X$ there is a unique ordinal $\alpha$ such that $x \in X^{\alpha} \backslash X^{\alpha+1}$, that is, such that $x$ is isolated in $X^{\alpha}$. Since $X$ is zero-dimensional, there exists then a clopen neighborhood $V_{x}$ of $X$ such that $V_{x} \cap X^{\alpha}=\{x\}$. Define a function $f_{x}$ from $X$ onto the two-element discrete space $\{0,1\}$ by $f_{x}\left(V_{x}\right)=\{1\}$ and $f_{x}\left(X \backslash V_{x}\right)=\{0\}$. Thus to each $x$ in $X$ we have defined a two-valued continuous function $f_{x}$ on $X$. Let $F=\left\{f_{x} \mid x \in X\right\}$.

We now show that this family $F$ of functions on $X$ separates points of $X$. Let $x$ and $y$ be any two distinct elements of $X$. Now two cases are possible. There may exist an $\alpha$ such that both $x$ and $y$ are isolated in $X^{\alpha}$ or there may not. If the first case holds then the function $f_{x}$ separates $x$ and $y$. For, $f_{x}(x)=1$ and $f_{x}(y)=0$. In the latter case, there exists a least ordinal $\alpha$ such that only one of the two elements belongs to $X^{\alpha+1}$, say $x$. Then $y \in X^{\alpha} \mid X^{\alpha+1}$ and $x \in X^{\alpha+1}$. Now $V_{y} \cap X^{\alpha}=\{y\}$. Therefore $x \notin V_{y}$ and so $f_{y}(x)=1$, but $f_{y}(y)=0$. Thus $f_{y}$ separates $x$ and $y$. Thus we have proved that $F$ separates elements of $X$.

Define a map $e: X \rightarrow\{0,1\}^{F}$ by $(e(x))_{f_{y}}=f_{y}(x) \forall x \in X$ and $\forall f_{y} \in F$. Clearly the map $e$ is continuous. It is one-to-one since $F$ separates points of $X$. Let $Y$ be the range of $e$. Then $Y$ is obviously zero-dimensional and Hausdorff. Also,

$$
\text { the weight of } \begin{aligned}
Y \text { is } & \leqq \text { the weight of }\{0,1\}^{F} \\
& =\text { cardinality of } F \\
& =\text { cardinality of } X \\
& =\text { cardinality of } Y .
\end{aligned}
$$

We complete the proof by showing that this map $e$ has the property that $e\left(X^{\alpha}\right)=Y^{\alpha}$ for each ordinal $\alpha$. 
We use transfinite induction on $\alpha$. To prove the result for $\alpha=1$, we show that $\epsilon$ takes isolated points to isolated points.

If $x \in X$ is isolated, then $V_{x}$ must be $\{x\}$ and so $f_{x}$ vanishes at all points other than $x$. It follows that $e(x)$ has nonzero entry only in the coordinate corresponding to $f_{x}$ and that $e(y)$ has zero entry there for all other elements $y$. Consequently $e(x)$ is an isolated point of $Y$. Conversely, if $x$ is a nonisolated point of $X$, then $e(x)$ is also nonisolated in $Y$, since $e$ is one-to-one and continuous. Thus $e\left(X^{\prime}\right)=Y^{\prime}$.

Suppose as induction hypothesis that we have proved that $e\left(X^{\beta}\right)=Y^{\beta}$ for each $\beta<\alpha$. If $\alpha$ is limiting, then

$$
\begin{aligned}
e\left(X^{\alpha}\right) & =e\left(\bigcap_{\beta<\alpha} X^{\beta}\right)=\bigcap_{\beta<\alpha} e\left(X^{\beta}\right) \quad(\text { since } e \text { is } 1-1) \\
& =\bigcap_{\beta<\alpha} Y^{\beta} \quad \text { (by induction hypothesis) } \\
& =Y^{\alpha} .
\end{aligned}
$$

Now let $\alpha$ be nonlimiting. Then since $e$ is $1-1$ and continuous, we have

$$
e\left(X^{\alpha}\right)=e\left(X^{\alpha-1+1}\right) \subseteq\left(e\left(X^{\alpha-1}\right)\right)^{1}=Y^{\alpha-1+1}=Y^{\alpha} .
$$

To prove the reverse inequality, we show that $e\left(X \backslash X^{\alpha}\right) \subset Y \backslash Y^{\alpha}$. Now

But

$$
e\left(X \backslash X^{\alpha}\right)=e\left(X \backslash X^{\alpha-1}\right) \cup e\left(X^{\alpha-1} \backslash X^{\alpha}\right) .
$$

$$
\begin{aligned}
e\left(X \backslash X^{\alpha-1}\right) & \subset Y \backslash Y^{\alpha-1} \quad \text { (by the induction hypothesis and since } e \text { is 1-1) } \\
& \subset Y \backslash Y^{\alpha} .
\end{aligned}
$$

If $x \in X^{\alpha-1} \mid X^{\alpha}$, then $x$ is isolated in $X^{\alpha-1}$ and so $f_{x}(x)=1$ and $f_{x}(y)=0$ for each $y \neq x$ in $X^{\alpha-1}$. Therefore $e(x)$ is isolated in $e\left(X^{\alpha-1}\right)=Y^{\alpha-1}$. Thus $e(x) \in Y^{\alpha-1} \backslash Y^{\dot{\alpha}}$.

Thus we have shown that $e\left(X^{\alpha}\right)=Y^{\alpha}$ for every ordinal $\alpha$. This in particular proves that $Y$ is scattered and the proof of the theorem is complete.

Corollary 6. Let $(X, \tau)$ be minimal (in the lattice of all topologies on $X)$ with respect to the property of being in $\mathscr{Z}$. Then the weight of $(X, \tau)$ is $\leqq$ the cardinality of $X$.

COROLlaRY 7. For each ordinal $\alpha$, let $\mathscr{Z}_{\alpha}$ denote the class of all members $(X, \tau)$ of $\mathscr{Z}$ such that $w(X)=\alpha$. If $(X, \tau)$ is minimal with respect to the property of being in $\mathscr{Z}_{\alpha}$, then the weight of $X$ does not exceed the cardinality of $X$.

COROLlary 8. Let $X$ be a countable space in $\mathscr{Z}$. Then there is a coarser topology on $X$ which is metrisable and scattered. 
The last corollary follows from Theorem 5 and the fact that every regular second-countable space is metrisable.

THEOREM 9. Let $X$ be a subspace of a well-ordered space. Then $X$ admits a weaker compact Hausdorff topology.

Proof. Let $\alpha=\sup X$. Define a map $g: X \rightarrow[1, \alpha]$ as follows:

$$
\begin{aligned}
g(x)=y & \text { if } \exists y \in \bar{X} \backslash X \text { such that } y<x \text { and }[y, x] \cap X=\{x\}, \\
& =\alpha \quad \text { if } \alpha \notin X \text { and if } x \text { is the least element of } X \\
& =x \quad \text { otherwise. }
\end{aligned}
$$

If $y_{1}$ and $y_{2}$ are two elements of $\bar{X} \backslash X$ such that $y_{1}<y_{2}<x$, it follows that $\left[y_{1}, x\right] \cap X$ must contain $y_{2}$ in its closure. So, the above function $g$ is well defined.

We claim that $g$ is one-to-one. Let $g\left(x_{1}\right)=g\left(x_{2}\right)$. If this common value is $\alpha$, then either $\alpha \in X$ in which case $x_{1}=x_{2}=\alpha$, or $\alpha \notin X$ in which case $x_{1}=$ $x_{2}=$ the least element of $X$. If this common value is $y \neq \alpha$, then either $y \in X$ in which case $x_{1}=x_{2}=y$, or $y \notin X$ in which case

$$
x_{1}=x_{2}=\operatorname{glb}\{x \in X \mid x \geqq y\} \text {. }
$$

Next we assert that if $x_{1} \leqq x_{2}$ in $X$, then $x_{1} \leqq g\left(x_{2}\right) \leqq x_{2}$, provided none of these points is the least element of $X$. This is immediate from the definition of $g$. This observation leads to two important consequences: First, except for the least element of $X$, the function $g$ is strictly order preserving. Secondly, if $\left(x_{\beta}\right)$ is an increasing transfinite well-ordered sequence in $X$ converging to $x$ in $X$, then $x_{\beta-1} \leqq g\left(x_{\beta}\right) \leqq x_{\beta}$ for each nonlimiting ordinal $\beta$ and so $\left(g\left(x_{\beta}\right)\right) \rightarrow x$. This shows that the map $g$ is continuous.

Finally, we show that the range of $g$ is closed in $[1, \alpha]$. Let $\left(g\left(x_{\beta}\right)\right) \rightarrow t$ be a well-ordered strictly increasing transfinite sequence in $g(X)$ converging to $t$ in $[1, \alpha]$. We want to show that $t \in g(X)$. If $g\left(x_{\beta_{1}}\right) \leqq g\left(x_{\beta_{2}}\right)$, we easily see that $g\left(x_{\beta_{1}}\right) \leqq x_{\beta_{1}} \leqq g\left(x_{\beta_{2}}\right)$. Therefore the transfinite sequence $\left(x_{\beta}\right)$ also converges to $t$ in $[1, \alpha]$. Therefore $t \in \bar{X}$.

If $t=\alpha$ and if $\alpha \in X$, then $g(\alpha)=t$ so that $t \in g(X)$; if $t=\alpha$ and if $\alpha \notin X$, then $g\left(x_{0}\right)=t$ where $x_{0}$ is the least element of $X$, so that $t \in g(X)$.

Let $t \neq \alpha$. If $t \in X$ and if $x_{1}<t$ is another element of $X$, then there exists $x_{2} \in X$ such that $x_{1}<x_{2}<t$ (since $t$ belongs to $\bar{X}$ ). This implies that $g(t)=t$ so that $t \in g(X)$. If $t \notin X$, then let $x=\operatorname{glb}\{y \in X \mid y \geqq t\}$. Then $t=g(x)$, so that $t \in g(X)$.

Thus $g(X)$ is a closed subset of $[1, \alpha]$.

Thus $g$ is a 1-1 continuous map from $X$ onto a compact Hausdorff space. 
COROLlARY 10 (KATETOV [3]). A countable regular space admits a weaker compact Hausdorff topology if and only if it is scattered.

Proof. One part follows easily from the facts that every countable compact Hausdorff space is scattered and that any topology finer than a scattered topology must be scattered. To prove the converse, let $X$ be a countable regular scattered space. Then $X \in \mathscr{Z}$. Therefore by Corollary 8 , there is a weaker topology which is metrisable and scattered. Call this new space $X_{1}$. Then by Corollary 3, $X_{1}$ is homeomorphic to a subspace of $[1, \Omega)$. Again by Theorem 9 , this subspace of $[1, \Omega)$ admits a weaker compact Hausdorff topology. It follows that $X$ admits a weaker compact Hausdorff topology.

\section{REFERENCES}

1. V. K. Bel'nov, On condensations onto compacta, Dokl. Akad. Nauk SSSR 193 (1970), 506-509=Soviet Math. Dokl. 11 (1970), 949-952. MR 42 \#3756.

2. V. Kannan and M. Rajagopalan, Countable topological spaces, Technical Publications of the Department of Mathematics, Madurai University (to appear).

3. M. Katetov, On the mappings of countable spaces, Colloq. Math. 2 (1949), 30-33. MR 12, 627.

4. B. Knaster and K. Urbanik, Sur les espaces complets séparables de dimension 0 , Fund. Math. 40 (1953), 194-202. MR 15, 641.

5. S. Mazurkiewicz and W. Sierpinski, Contribution à la topologies des ensembles d'enombrables, Fund. Math. 1 (1920), 17-27.

6. S. Mrowka, M. Rajagopalan and T. Soundararajan, A characterisation of compact scattered spaces through chain limits (Chain compact spaces), Proc. Pittsburgh Conference in Topology II (1972), Academic Press, New York (to appear).

7. R. S. Pierce, Existence and uniqueness theorems for extensions of zero-dimensional compact metric spaces, Trans. Amer. Math. Soc. 148 (1970), 1-21. MR 40 \#8011.

8. C. Ryll-Nardzewski and R. Telgarsky, On scattered compactification, Bull. Acad. Polon. Sci. Sér. Sci. Math. Astronom. Phys. 18 (1970), 233-234. MR 41 \#7635.

9. Z. Semadeni, Sur les ensembles clairsemés, Rozprawy Math. 19 (1959), 39 pp. MR 21 \#6571.

10. W. Sierpinski, Sur une propriété topologique des ensembles denombrables denses en soi, Fund. Math. 1 (1920), 11-16.

Department of Mathematics, Madurai University, Madurai, India

Department of Mathematics, Memphis State University, Memphis, Tennessee 38111 